\title{
Cardiovascular Diseases
}

CASE REPORT

\section{Coronary Artery Ectasia: An Interventional Cardiologist's Dilemma}

\author{
Matthew Schmidt and Timothy E Paterick*
}

Bay Care Medical Group, Green Bay, Wisconsin, USA

*Corresponding author: Timothy E Paterick, MD, JD. MBA, Bay Care Clinic, 4950 Founders Terrace, Hobart, Wisconsin 55145, USA, E-mail: tpaterick@gmail.com

\begin{abstract}
Coronary artery ectasia is defined as a localized, or diffuse dilation of a coronary artery lumen. Coronary artery ectasia is well recognized, but a rare finding encountered during diagnostic coronary angiography. Coronary artery ectasia represents a form of atherosclerotic coronary artery disease, seen in $1.4-4.9 \%$ of patients undergoing coronary angiography. It may be an isolated finding, or in combination with stenotic lesions.

The classification of coronary artery ectasia is divided into four groups: Type 1: Diffuse ectasia of two or three vessels, Type 2: Diffuse ectasia in one vessel and localized disease in another vessel, Type 3: Diffuse ectasia in one vessel only, and Type 4: Localized or segmental involvement.

This case illuminates the difficult decision making regarding stenting of coronary arteries with ectasia and atherosclerosis. The additional challenge is whether the benefit of anticoagulation with warfarin outweighs the bleeding risk. The treatment approach is often ambiguous, and can be a vexing clinical question, as identified in our case presentation.
\end{abstract}

\section{Keywords}

Coronary artery ectasia, Coronary aneurysm, Thrombus, Stenting, Anticoagulation

\section{Introduction}

Coronary artery ectasia (CAE) is defined as a localized, or diffuse dilation of the coronary artery lumen exceeding the largest diameter of an adjacent normal coronary vessel by 1.5 fold. CAE is well recognized, but an uncommon finding encountered during diagnostic coronary angiography. The classification of CAE is divided into four groups: Type 1: Diffuse ectasia of two or three vessels, Type 2: Diffuse ectasia in one vessel and localized disease in another vessel, Type 3: Diffuse ectasia in one vessel only, and Type 4: Localized or segmental involvement [1].
The term 'ectasia' refers to diffuse dilation of a coronary artery, while focal coronary dilation is called 'a coronary aneurysm' [2]. The exact pathophysiology of CAE is unknown. CAE is an anatomical variant and a phenotypic expression of coronary artery disease that may present with myocardial ischemia or coronary syndrome. The incidence varies between 1.2-4.9\% [3]. The CASS registry found CAE in $4.9 \%$ of coronary angiograms [3]. Our group classifies CAE as small (vessel size $<5$ $\mathrm{mm}$ ), medium (vessels size 5-8 $\mathrm{mm}$ ) and giant (vessel size $>8 \mathrm{~m}$ ). CAE is defined as a coronary artery lumen dilatation with a diameter of 1.5 times the adjacent normal coronary artery based on CASS registry [3].

\section{Etiology}

The etiology of CAE can be enigmatic. Atherosclerosis is considered the principal etiologic cause responsible for greater than $50 \%$ of cases in adults, while Kawasaki disease is the most common cause in children and young adults [4]. The histology of atherosclerosis and ectasia are comparable. The mechanism of luminal dilation in some atherosclerotic vessels is ambiguous; atherosclerosis typically causes a narrowing of the vessel lumen. Luminal dilation due to arterial remodeling of certain plaques results in expansion of the media and external elastic membrane. This proposed methodology of arterial remodeling may be operative in the case of CAE.

Intravascular ultrasound reveals that arterial remodeling can be bidirectional depending upon expansion or contraction of the external elastic membrane. CAE is believed to be exaggerated expansive remodeling of the external elastic membrane resulting in luminal expansion. Enzymatic degradation of the extracellular matrix-by-matrix metalloproteinases and other lytic en-

\footnotetext{
Citation: Schmidt M, Paterick TE (2018) Coronary Artery Ectasia: An Interventional Cardiologist's Dilemma. Int Arch Cardiovasc Dis 2:007

Accepted: July 26, 2018; Published: July 28, 2018

Copyright: (c) 2018 Schmidt M, et al. This is an open-access article distributed under the terms of the Creative Commons Attribution License, which permits unrestricted use, distribution, and reproduction in any medium, provided the original author and source are credited.
} 
zymes and thinning of the tunica media associated with severe chronic inflammation is considered the principal pathogenesis of the expansive remodeling [5]. CAE is also related to apical HCM with high wall tension. Formation of aneurysms may be seen post percutaneous coronary interventions including balloon angioplasty, stent placement, and atherectomy. The mechanism is felt to be injury of the media of the blood vessel [6].

\section{Diagnosis}

Coronary angiography is the gold standard in diagnosing CAE. Intravascular ultrasound (IVUS) is critical to the evaluation of luminal characteristics and pathologies [6]. Distortions in coronary flow and washout are common in CAE and are related to the severity of ectasia. Signs of stagnant flow include: Delayed antegrade contrast filling, segmental back flow, and stasis in the ectatic coronary segment [5].

\section{Clinical Significance}

Symptoms may be associated with concomitant coronary disease, Kawasaki disease, or connective tissue disease. Most patients are asymptomatic. Patients with CAE may present with angina, positive stress tests, and acute coronary syndrome. Diminished coronary flow, or stagnancy of blood flow, may cause exercise-induced angina without coexistent stenotic coronary artery disease. Formation of intracoronary thrombus and dissipation of emboli distally may be the trigger of the acute coronary syndrome, which is hastened by stagnant flow in the ectatic coronary segment. CAE is predisposed to vasospasm, which may elicit angina, or acute coronary syndrome. In people less than 50 years CAE should raise a concern for connective tissue disorders and vasculitides $[7,8]$.

\section{Prognosis}

Prognosis for CAE is directly related to the severity of concomitant coronary artery disease. CAE with underly- ing coronary artery disease is a malicious combination with an increased potential for adverse cardiac events. Isolated CAE still carries the risk of myocardial ischemia and infarction. Type 1 and Type 2 CAE carry a higher risk than Type 3 and Type 4 CAE. There is no reported data showing a relationship between the diameter of an artery and outcome [9].

\section{Treatment}

Management of CAE is fraught with uncertainty because the rarity of CAE prevents large randomized trials comparing different treatment approaches. When CAD coexists intense primary and secondary risk factor modifications is mandatory.

Management of isolated CAE in the case with angina or myocardial ischemia includes ASA, statin, and anti-ischemic medications. Acute coronary syndromes associated with CAE may require thrombolysis, heparin administration and glycoprotein $2 \mathrm{~b} / 3$ a receptor inhibitors. Thrombus aspiration may be needed during primary $\mathrm{PCl}$. Percutaneous and surgical interventions are often needed in patients with CAE and stenotic lesions where angina persists despite maximal medical therapy. Optimal stent sizing, misplacement, and embolization of stents, early stent thrombosis, and restenosis haunt stenting procedures $[10,11]$.

Long-term anticoagulation is a debated topic with no randomized trials to confer benefit. The antiplatelet benefit must be weighed against the risk of hemorrhage.

\section{Case}

51-year-old African American male presented to the emergency room with chest pain. He was found to have an elevated troponin. The blood troponin level was 0.45 $(<0.05)$ and rose to $2.6 \mathrm{His}$ ECG was abnormal. (Figure 1) The ECG was unchanged from the one recorded in 2014.

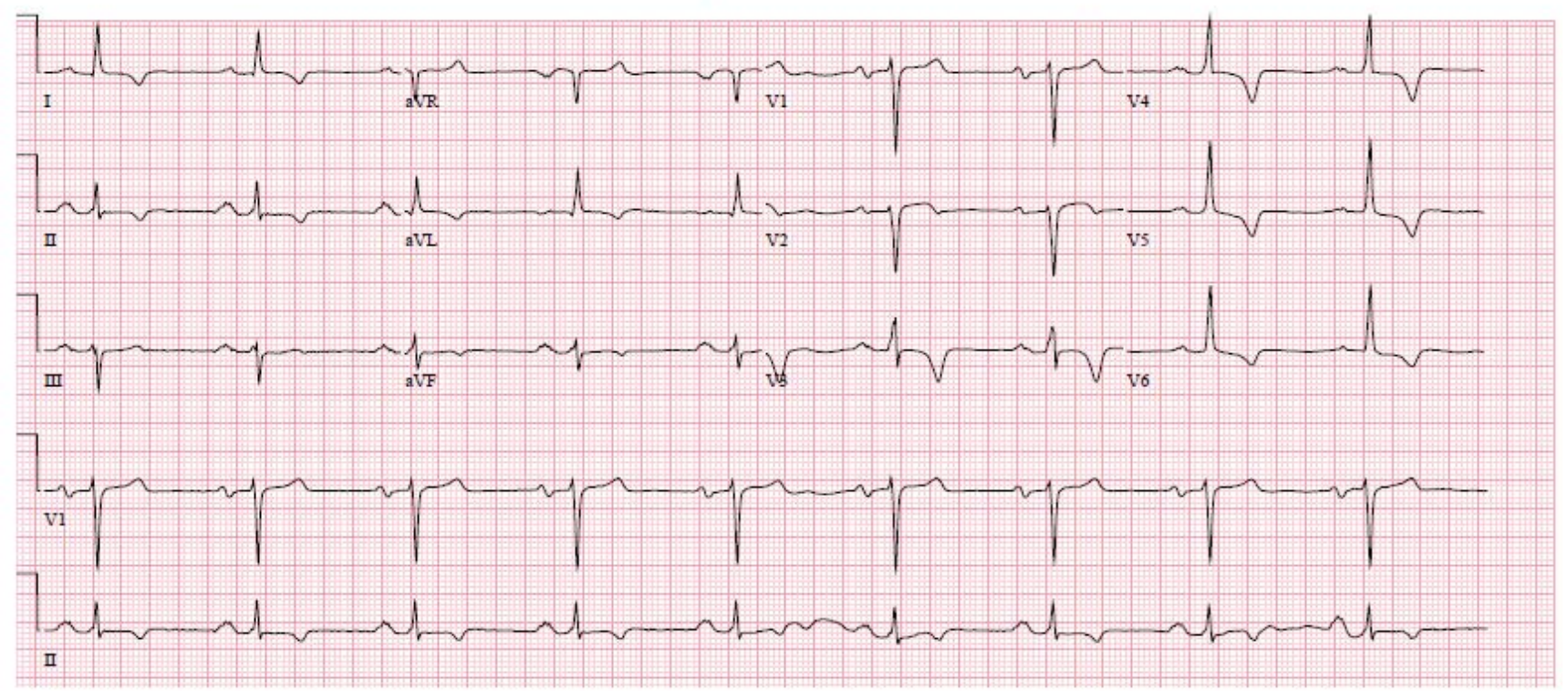

Figure 1: Sinus rhythm, left axis, and inverted t waves 1, 2 aVL, V 3-6. 


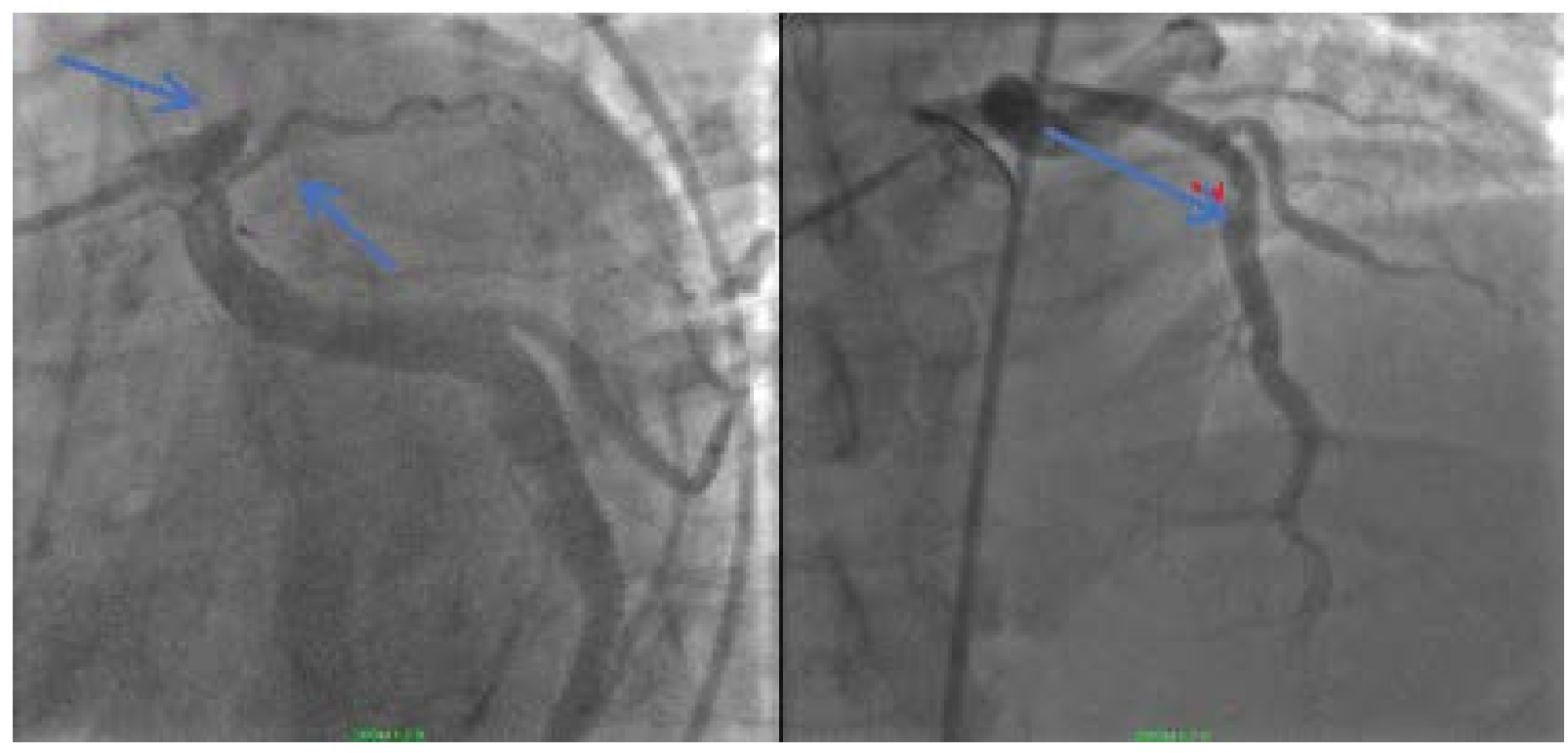

Figure 2: a) Coronary angiogram LAO view revealing occluded proximal LAD (2014); b) Coronary angiogram RAO post thrombectomy and placement of a $4.0 * 22$ resolute stent (2014).

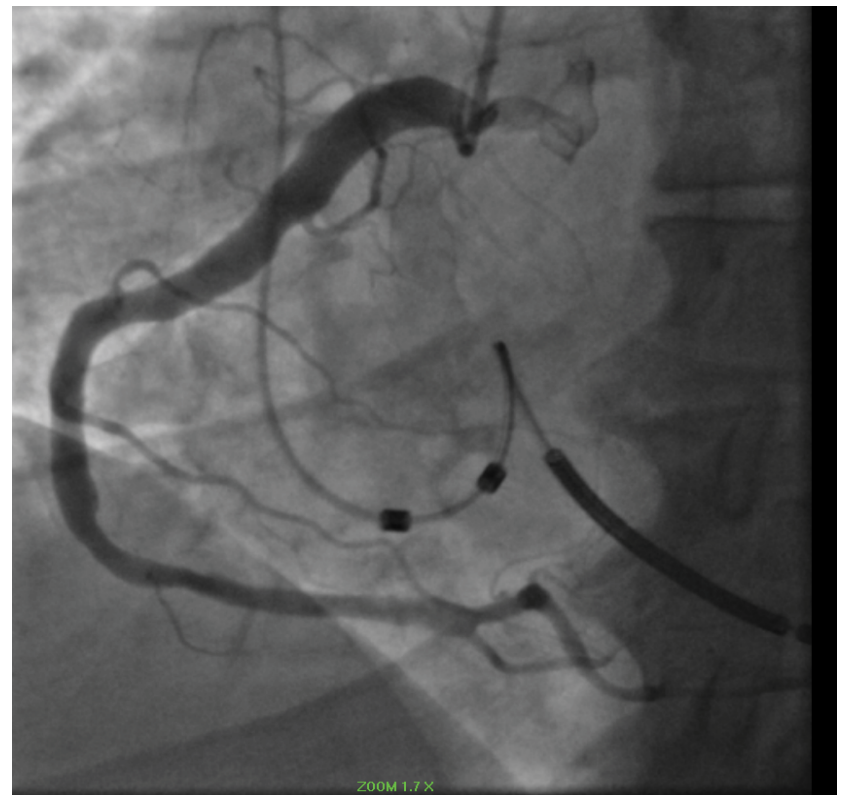

Figure 3: Ectatic right coronary artery.

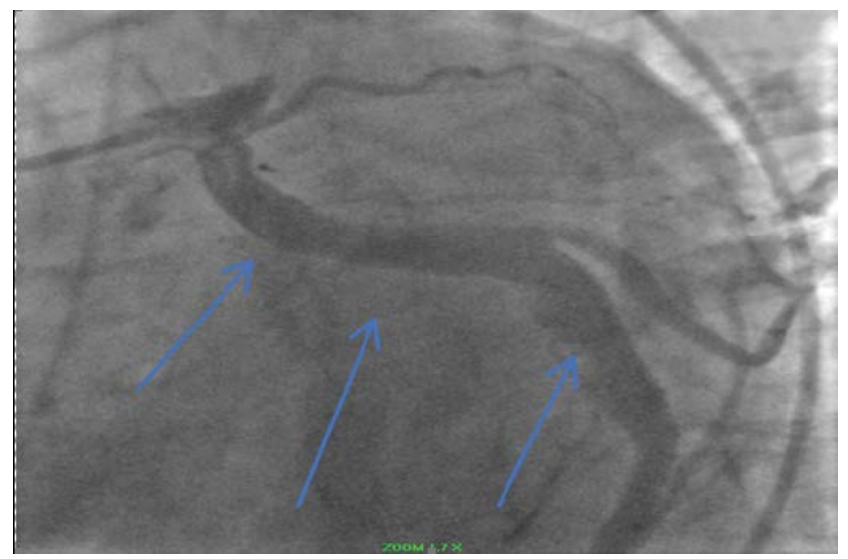

Figure 4: Ectatic circumflex coronary artery (Blue arrows).

Four years previously the patient had experienced an acute occlusion of his proximal left anterior descend-

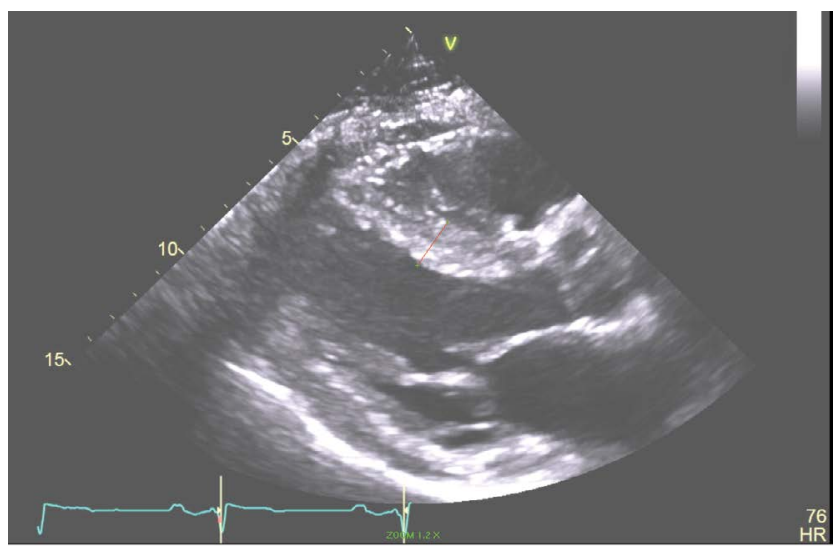

Figure 5: The Parasternal Long axis of the LV revealing a $17 \mathrm{~mm}$ septum in a patient with $\mathrm{HCM}$.

ing coronary artery (LAD). He underwent thrombectomy and proximal LAD stenting (Figure $2 \mathrm{a}$ and Figure $2 \mathrm{~b}$ ).

During angiography Type 1 CAE was identified. The right coronary artery (RCA), Circumflex coronary artery (Cf) and LAD were all large luminal vessels consistent with coronary artery ectasia. (Figure 3 and Figure 4) The patient had a symptomatic episode of ventricular tachycardia that was hemodynamically compromising and was managed with cardioversion. The patient recovered and was treated with ASA and ticagrelor for 18 months and then changed to just ASA. He remained active and free of cardiovascular symptoms.

Past Medical History: The patient has a history of gene-negative hypertrophic cardiomyopathy (HCM). In 1983 at age 16 years he had a syncopal episode while playing basketball and was identified to have HCM by echocardiography (Figure 5, Figure 6, Figure 7 and Figure 8). In 2002 the patient had another syncopal episode was found to have non-sustained ventricular tachycardia on Holter monitoring. He had an ICD placed. He had hypertension, coronary artery disease, $\mathrm{HCM}$, hyperlipid- 


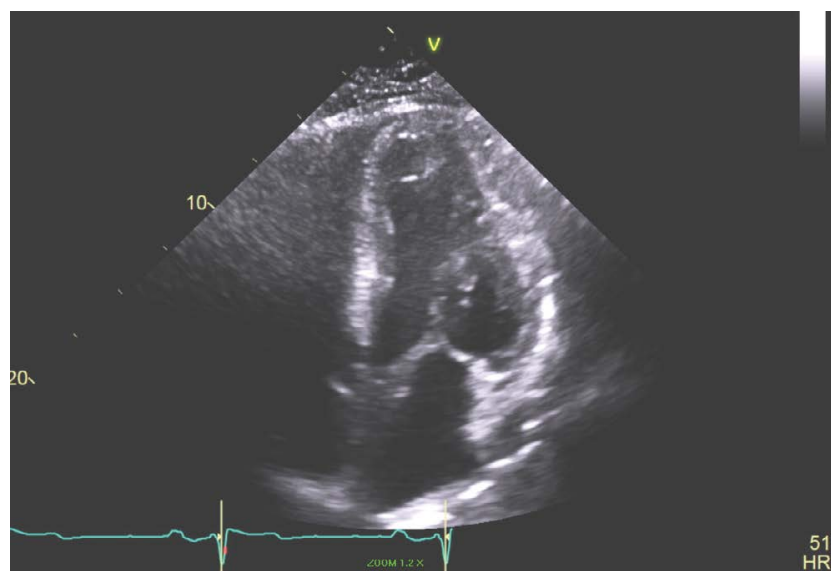

Figure 6: Apical 4 chamber view of the LV showing small LV cavity.

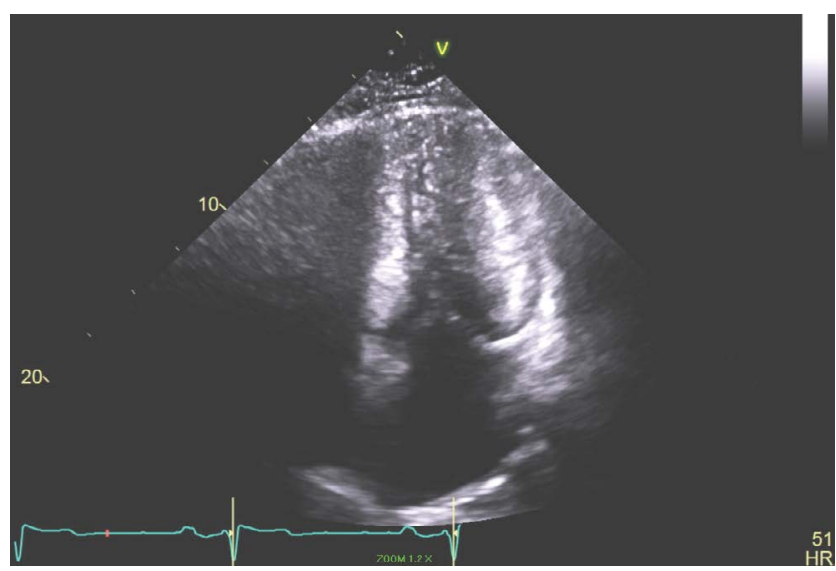

Figure 7: Apical 4 chamber view of the LV showing the end systolic dimension with LV cavity obliteration.

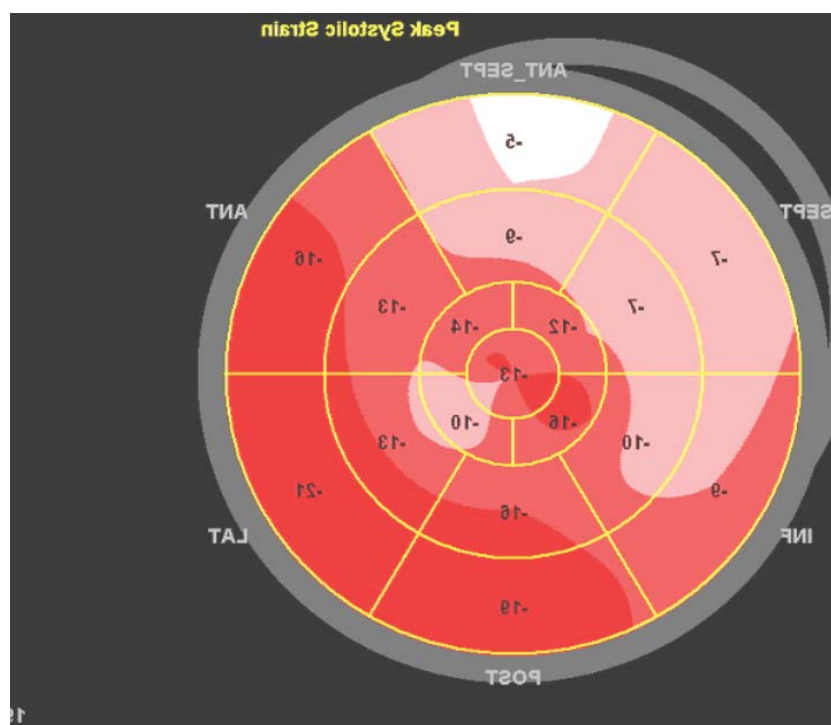

Figure 8: Peak systolic strain of the LV revealing diminishes anterior, mid, and inferior septal strain.

emia, syncope, NSVT and post-myocardial infarction VT that was managed with shock therapy.

Family History: Was positive for premature $C A D$ with his father dying of a myocardial infarction at age 56 years and his mother having coronary bypass at age 56 years.

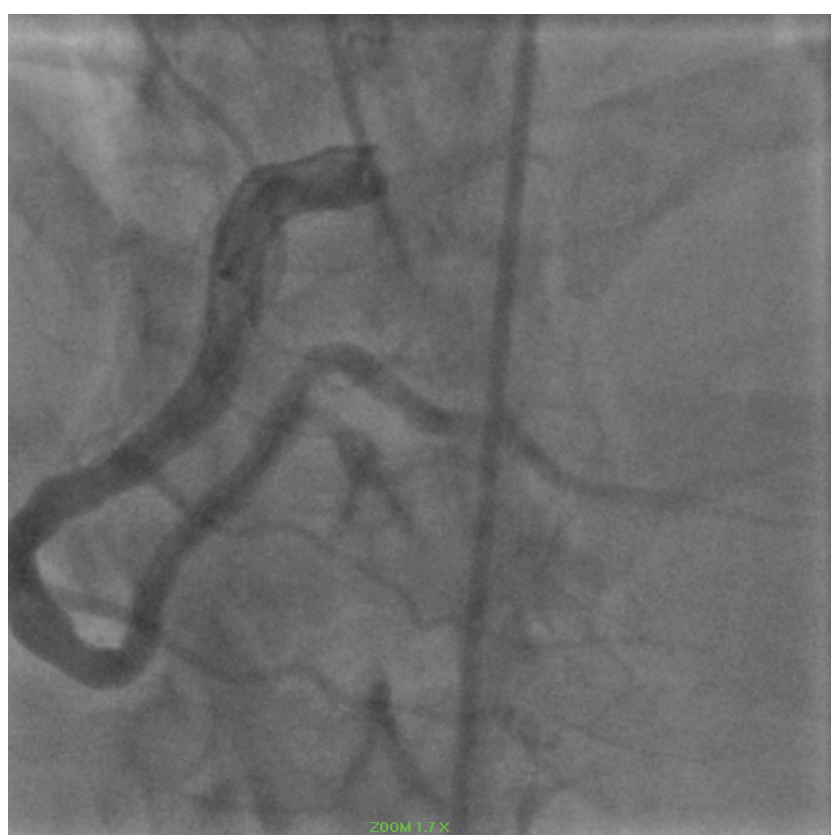

Figure 9: Ectatic RCA.

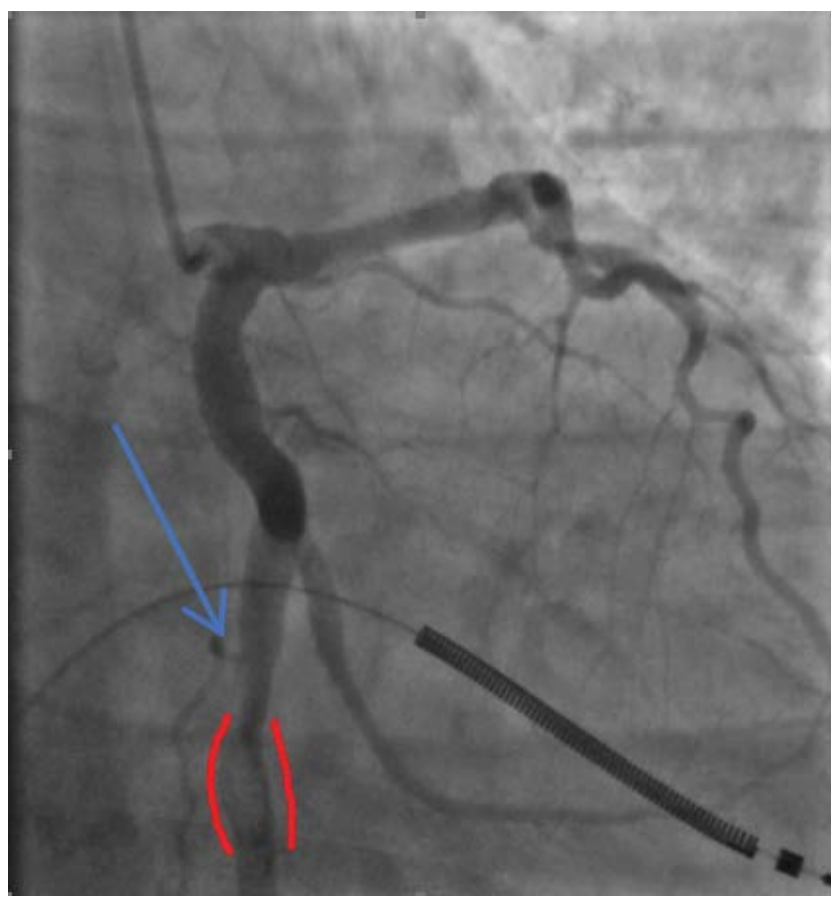

Figure 10: Circumflex Thrombus: red outline 2018.

Physical Examination: Reveals a well developed black male with a blood pressure $130 / 70 \mathrm{mmHg}$ and pulse of $48 \mathrm{bpm}$. JVP: normal, carotid upstroke: Normal S1, S2, S4, and 1/6 systolic ejection murmur, and palpable upper and lower extremity pulses.

The 2018 presentation was consistent with a NSTEMI. Coronary angiography revealed a patent RCA (Figure 9).

Thrombus was identified in an ectatic circumflex coronary artery (Figure 10).

Thrombus was identified in the LAD (Figure 11).

The patient was treated for 72 hours with aspirin and ticagrelor. The hope was to dissolve the thrombus and 


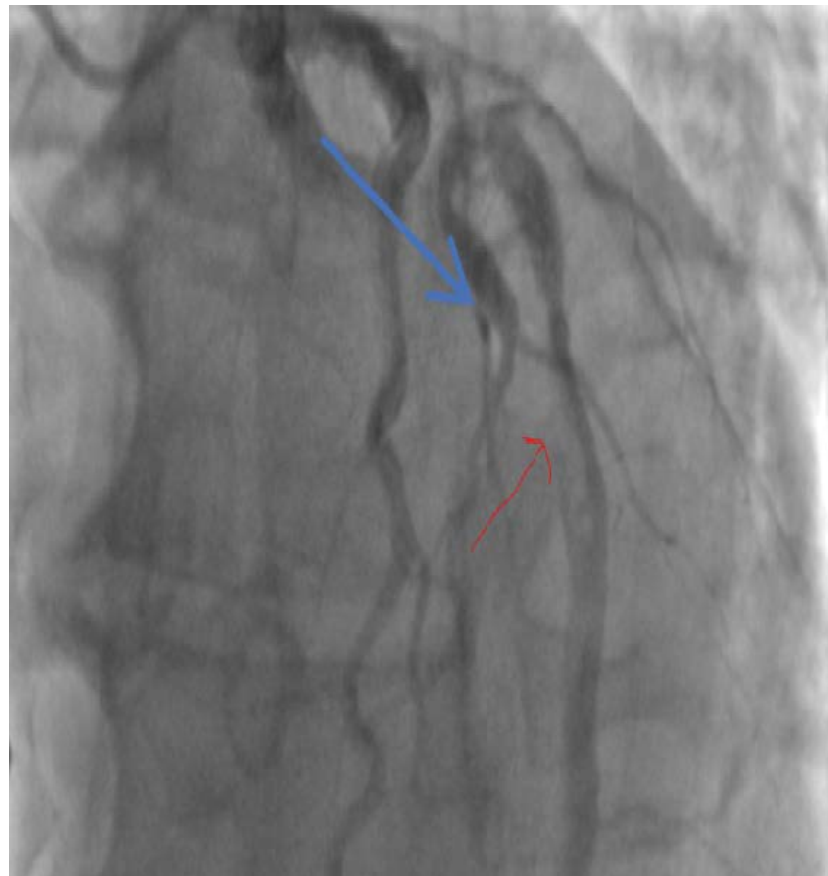

Figure 11: LAD clot (blue arrow) 2018.

determine the degree of underlying atherosclerosis.

Follow up angiography revealed that the thrombus burden in the circumflex and LAD was unchanged. The patient had no further clinical symptoms. Aspirin was continued and heparin was initiated. Additionally, the patient was started on warfarin therapy after discussing the risks and benefits. Once the INR was 2.5 the patient was discharged to follow up.

\section{Discussion}

Treatment options for CAE include risk factor modification, anti-ischemic therapy, ASA, and antithrombotic therapy. Many authors recommend chronic anticoagulation, however; no randomized trial demonstrates its benefit in CAE. The anticipated benefit must be counterbalanced against the risk of hemorrhage.

In this case, the recurrent thrombus formation in Type 1 ectatic coronary arteries placed the patient at high risk for recurrent ischemia and myocardial infarction. The high-risk profile of this patient made the acknowledgment of bleeding complications from warfarin therapy acceptable. The patient was discharged with a plan for careful monitoring of his INR and clinical symptoms. A nuclear stress test was arranged for 6 weeks.

This case elucidates the challenging decision-making associated with stenting of coronary arteries with ectasia and atherosclerosis and using warfarin for anticoag- ulation to prevent coronary thrombus formation. The treatment of coronary artery ectasia with atherosclerosis is a vexing clinical question as identified in our case presentation. Presently these decisions must be made on an individualized basis.

\section{Conflicts of Interest}

There are no conflicts of interest.

\section{Acknowledgement}

No acknowledgements.

\section{References}

1. AR Zeina, D Sharif, J Blinder, Rosenschein U, Barmeir E (2007) Noninvasive assessment of coronary artery using multidetector computed tomography. Coron Artery Dis 18: 175-180.

2. S Mavrogeni (2010) Coronary artery ectasia: From diagnosis to treatment. Hellenic J Cardiol 51: 158-163.

3. PS Swaye, LD Fisher, P Litwin, PA Vignola, MP Judkins, et al. (1983) Aneurysmal coronary artery disease. Circulation 67: $134-138$.

4. Díaz-Zamudio $M$, Bacilio-Pérez $U$, Herrera-Zarza MC, Meave-González A, Alexanderson-Rosas E, et al. (2009) Coronary artery aneurysms and ectasia: Role of coronary CT angiography. Radiographics 29: 1939-1954.

5. AP Antoniadis, YS Chatzizsis, GD Giannoglou (2008) Pathogenetic mechanisms of coronary ectasia. Int J Cardiol 130: 335-343.

6. A Magginas, DV Cokkinos (2006) Coronary artery ectasias: Imaging, functional assessment and clinical implications. Eur Heart J 27: 1026-1031.

7. Sayin $T$, Döven $O$, Berkalp $B$, Akyürek $O$, Güleç $S$, et al. (2001) Exercise-induced myocardial ischemia in patients with coronary artery ectasia without obstructive coronary artery disease. Int J Cardiol 78: 143-149.

8. Zografos TA, Korovesis S, Giazitzoglou E, Kokladi M, Venetsanakos I, et al. (2013) Clinical and angiographic characteristics of patients with coronary artery ectasia. Int $\mathrm{J}$ Cardiol 167: 1536-1541.

9. S Gulec, Y Atmaca, M Kilickap, Akyürek O, Aras O, et al. (2003) Angiographic assessment of myocardial perfusion in patients with isolated coronary artery ectasia. Am J Cardiol 91: 996-999.

10. H Turnhan, E Yetkin (2007) What is the plausible strategy for the management of patients with isolated coronary ectasia and myocardial ischemia? Int J Cardiol 117: 285-286.

11. Levine GN, Bates ER, Blankenship JC, Bailey SR, Bittl JA, et al. (2011) 2011 ACCF/AHA/SCAI Guideline for Percutaneous Coronary Intervention: a report of the American College of Cardiology Foundation/American Heart Association Task Force on Practice Guidelines and the Society for Cardiovascular Angiography and Interventions. Circulation 124: e574-e651.

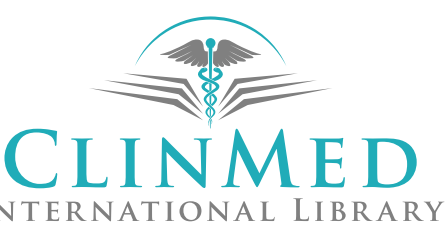

